\title{
Reducing Fontan effusions: A day saved is a dollar earned
}

\author{
Rozelle Corda, MSN, FNP-BC
}

\footnotetext{
From the Department of Congenital and Pediatric Cardiac Surgery, Division of Cardiothoracic Surgery, New York Presbyterian Morgan Stanley Children's Hospital of New York, Columbia University Medical Center, New York, NY; and the Columbia University School of Nursing, New York, NY.

Disclosures: Author has nothing to disclose with regard to commercial support.

Received for publication July 2, 2015; accepted for publication July 2, 2015; available ahead of print Aug 5, 2015. Address for reprints: Rozelle Corda, MSN, FNP-BC, Columbia University, Congenital and Pediatric Cardiac Surgery, New York Presbyterian Morgan Stanley Children's Hospital of New York, Columbia University Medical Center, 3959 Broadway, BH 275, New York, NY 10032 (E-mail: rc367@cumc.columbia.edu).

J Thorac Cardiovasc Surg 2015;150:488-9

$0022-5223 / \$ 36.00$

Copyright $(c) 2015$ by The American Association for Thoracic Surgery

http://dx.doi.org/10.1016/j.jtcvs.2015.07.006
}

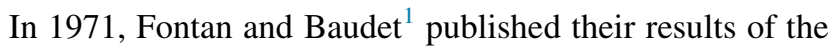
first physiologic correction for tricuspid atresia. The 2 survivors, despite being "anatomically and hemodynamically privileged," in that they had adequately sized pulmonary arteries with low pressures, had a postoperative course complicated by pleural effusions. In 1983, also despite operating on ideal candidates in their 9-year experience with 24 patients undergoing physiologic correction with the classic Fontan procedure, Ottenkamp and coworkers ${ }^{2}$ reported the occurrence of pleural effusion. In the following decades, changes in surgical strategies and techniques were introduced, and improvements in survival occurred; however, the morbidity of pleural effusions persisted. Many attempts have been made to reduce the prolonged duration of chylous and nonchylous effusion, yet it continues to be a longstanding, multifactorial problem without an easy answer. It is still a work in progress.

In attempt to standardize medical practice patterns, Cava and colleagues ${ }^{3}$ in 2005 devised a regimen with several treatment tactics tailored to this specific population. Commonly referred to as the Wisconsin protocol, the course of care incorporates specific diuretics and fluid restrictions, diet specifications, angiotensin-converting enzyme inhibition, and oxygen supplementation. Complications from effusions such as renal failure, thrombus formation, arrhythmias, rate of reintubation, and inotropic use were assessed. With the use of such consistent care strategies, the mean stay was reduced from 18 to 6 days, and chest tube duration was reduced from 15 to 6 days.

In their article in this issue of the Journal, Pike and colleagues ${ }^{4}$ compare hospital stay, duration of effusion, and readmission rates in 120 total patients at a single center before and after the implementation of a modified Wisconsin protocol. Although this preintervention and postintervention analysis is limited by a lack of data regarding specific preintervention clinical practice and compliance after the intervention, the article demonstrates that the use of a planned protocol resulted in decreased number of effusions greater than 1 week in

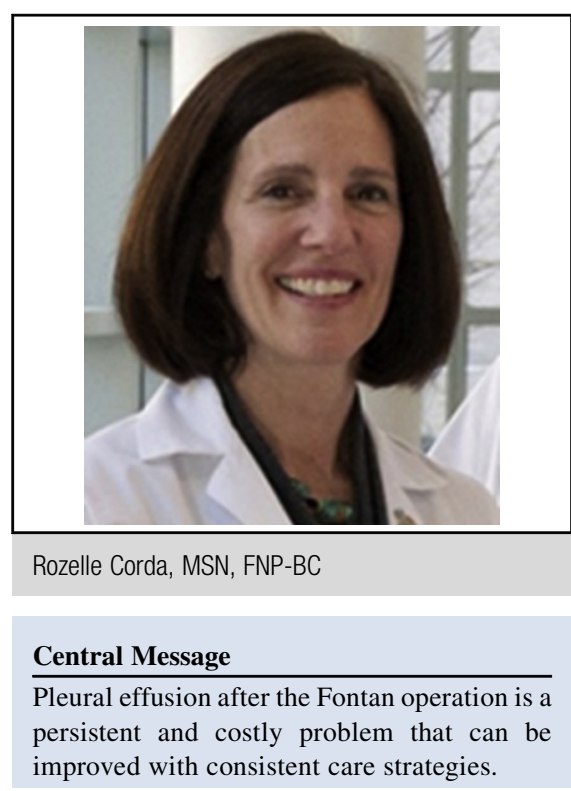

See Article page 481

duration, reduced hospitalization duration, and fewer readmissions. No patient in the postintervention group required the withholding of oral intake or total parenteral nutrition. The protocol described differs from the Wisconsin protocol with increased frequency of diuretics, warfarin administration, and the duration of a low-fat diet. One must consider that not all patients may require angiotensin-converting enzyme inhibition or supplemental oxygen until tubes are removed, and these guidelines do not define at what point further interventions, such as catheterization or duct ligation, would be helpful. Although these recommendations may certainly ensure efficiency, caution should be exercised to avoid being blindsided by forgetting to look for causal relationships to clinical problems beyond those commonly addressed by these plans.

Pike and colleagues ${ }^{4}$ go beyond identifying length of stay reductions to include a cost analysis according to the mean total costs in the preprotocol versus postprotocol groups, which makes this article more meaningful. They calculated a saving of \$9430/patient on hospital cost related to the Fontan procedure, and a saving of $\$ 5143 /$ patient related to the readmissions due to recurring symptomatic effusion. The 2007 discharge group was analyzed on the basis of cost of hospitalization figures derived from patients discharged in 2004. Extrapolating data from the National Health Expenditures Projections on annual hospital cost expenditure increases, ${ }^{5}$ one can loosely project the 2015 increase in savings for their hospital costs related to the Fontan operation 
to be greater than $\$ 14,000 /$ patient. Similarly, the saving related to readmission would be nearly $\$ 8000 /$ patient. Although these are gross projections, the implications are clear. It does remind us that the days when we could practice with the sole intention of patient care without regard to cost are gone. With more than $17 \%$ of the 2012 Gross Domestic Product in the United States spent on health care expenditures and $29 \%$ of those expenditures associated with inpatient hospital stays, ${ }^{6}$ we have been forced to become fiscally aware and responsible with regard to patient care.

It has been 47 years since Francis Fontan performed the first correction for tricuspid atresia. Despite almost 5 decades of practice, the involvement of hundreds of surgeons and thousands of patients with single-ventricle physiology, a few modifications, vast technologic advancements, and technical mastery, a persistent complication, pleural effusion, persists. Congratulations to Pike and colleagues ${ }^{4}$ for addressing the question of cost savings, which is an important element today. I am hopeful that before another half century goes by, with studies like these, we will have even better outcomes.

\section{References}

1. Fontan F, Baudet E. Surgical repair of tricuspid atresia. Thorax. 1971;26:240-8.

2. Ottenkamp J, Rohmer J, Quaegebeur J, Brom AG, Fontan F. Nine years' experience of physiological correction of tricuspid atresia: long-term results and current surgical approach. Thorax. 1998;37:718-26.

3. Cava JR, Bevandic SM, Steltzer MM, Tweddell JS. A medical strategy to reduce persistent chest tube drainage after the Fontan operation. Am J Cardiol. 2005;96 130-3.

4. Pike NA, Okuhara C, Toyama J, Gross B, Wells W, Starnes V. A modified Fontan management protocol reduces pleural drainage, length of stay, and readmissions. J Cardiovasc Thorac Surg. 2015;150:481-7.

5. Centers for Medicare and Medicaid Services. National healthcare expenditures 2011-2021 [Internet]. Woodlawn (MD): The Centers; 2015. Available at: http://www.cms.gov/Research-Statistics-Data-and-Systems/Statistics-Trendsand-Reports/NationalHealthExpendData/Downloads/Proj2011PDF.pdf. Accessed June 26, 2015.

6. Moore B, Levit K, Elixhauser A. Costs for hospital stays in the United States, 2012 Statistical Brief \#181. NCBI Bookshelf Healthcare Cost and Utilization Project Statistical Briefs [Internet]. Rockville (MD): Agency for Healthcare Research and Quality; 2014. Available at: http://www.hcup-us.ahrq.gov/reports/statbriefs/ sb181-Hospital-Costs-United-States-2012.pdf. Accessed June 26, 2015. 